\title{
Volunteer-led Free Dental Outreach Clinics in semi-urban Uganda: a feasibility study
}

Dr David Lawrence, MBChB, MSc ${ }^{1,2}$

Dr Rebecca Barr, BDS ${ }^{1}$

Mr Enoch Magala, BComm ${ }^{1}$

${ }^{1}$ The Centre for Youth Driven Development Initiatives, Ndazabazadde, Wakiso, Uganda

${ }^{2}$ Brighton and Sussex University Hospitals NHS Trust, Royal Sussex County Hospital, Eastern Road, Brighton, BN2 5BE

Declaration of interests: None declared

\section{Abstract:}

Background: The global burden of dental disease remains a key global public health issue, with the prevalence of common pathologies grossly unchanged over the last two decades. Poor oral health impacts individuals on a personal and economic level and incurs a significant cost globally. The role of dental aid organisations in strategies to deliver dental health services has long been debated.

Methods: We launched a dental clinic at the premises of an established non-governmental organization. All patients were taught a locally developed oral hygiene educational module before receiving a thorough examination. Treatments offered included simple scaling, fluoride application, treatment of infection and extraction.

Results: Three dental clinics were conducted. A total of 93 patients registered. 39 (42\%) were male, with an average age of 20 years (range $5-70$ years). $86 \%$ of patients presented in pain. 5 (5.4\%) of patients were found to have sound teeth. $43(46 \%)$ patients had at least one tooth extraction. $26 \%$ of patients were referred for restorations. The treatment provided equated to 178 Units of Dental Activity (UDAs). The total cost per patient was $£ 1.82$, regardless of treatment, or $£ 0.95$ per UDA.

Discussion: We demonstrated a distinct need for dental healthcare in this area, an acceptability of our service, potential for sustainability, and a dedication towards providing a Basic Package of Oral 
Care. We call for a reignition of debate regarding the role of dental aid organisations in the provision of global oral healthcare.

\section{Background:}

In 2010, untreated caries in permanent teeth was the most prevalent dental condition worldwide, affecting an estimated 2.4 billion people, with untreated caries in deciduous teeth affecting over 621 million children. Crucially, there is no evidence that the prevalence and incidence has changed in the last twenty years ${ }^{1}$. Alongside this it has been documented that dental caries have an appreciable impact on oral-health related quality of life of young people in African countries such as Uganda ${ }^{2}$. In addition to a reduced quality of life, living in chronic pain can have a personal financial impact: whilst it is estimated that the direct treatment cost due to dental diseases worldwide corresponds to $4.6 \%$ of global health expenditure it is also found that indirect costs, including loss of earnings, correspond to economic losses similar to those of the ten most frequent causes of death ${ }^{3}$. It has also been established that the cost of dental care and the distance one must travel to reach a service can be significant barriers to accessing treatment ${ }^{4}$. It is also clear that providing a greater number of services across a broader geographical region will lead to increased usage and improved dental health ${ }^{5}$.

The East African county of Uganda is not unique to the rest of the African continent in that it suffers the effects of poor dental health described above. The Government of Uganda commits to providing free dental care at government-run facilities throughout the country as part of the Uganda National Minimum Healthcare Package ${ }^{6}$. Only $0.1 \%$ of the Ugandan health budget (which itself is $9 \%$ of the national budget) is allocated to dental care which leaves a highly underfunded service with no option but to charge patients a nominal fee for treatment. Although some work can be provided by dental officers, there are currently only around 200 dentists for Uganda's growing population of over 37 million people and with these few being poorly paid by the government there is a tendency to supplement income with private work or leave the public sector entirely. This shortage of human resources for health is exacerbated by the fact that currently there is only one five-year BDS degree offered within the country?.

The collective term of Dental Aid Organisations (DAOs) is used to describe non-governmental organisations that focus on global dental health. Classically DAOs are small in size, rely 
predominantly on a volunteer workforce and have small budgets (below $\$ 100,000$ per year). They focus their activities around service provision, technical assistance, education, and training and do little in terms of policy and advocacy. DAOs report common struggles, predominantly with recruiting volunteers, funding their projects, and integrating their work into local health systems ${ }^{8}$.

Beyond the local, practical difficulties, there exists a body of literature which critiques the very concept of DAOs in the developing world. Dickson and Dickson outline the undesirable side effects as including, but not limited to; being unilateral and not providing any reciprocal opportunities for workers in developing countries to practise in developed countries; creating unrealistic expectations among local people that local dental workers will not be able to meet by providing advanced treatment using more modern equipment; highlighting the imperfections of the local health system; and co-facilitating the movement of skilled labour into developed countries (commonly referred to as the brain drain) ${ }^{9}$. Arguments that are more broadly applied to the concepts of international development and aid as a whole are also applied to the work of DAOs, such as issues related to sustainability, disregarding local systems, and creating NGO dependence ${ }^{10}$.

With an awareness of the above, our research team aimed to determine whether it was feasible to provide a free dental outreach clinic at the premises of an established Ugandan non-governmental organisation (NGO), utilising international and local volunteers to do so. By providing such a service we intended to; educate the community about general oral hygiene and health; understand the prevalence of various pathologies; assess the need for free dental care within a semi-urban community; learn if this would impact on pre-existing locally provided dental care and gain awareness of potential harms caused by such a service.

\section{Methods:}

We launched a dental clinic at the premises of an established non-governmental organization (NGO), The Centre for Youth Driven Development Initiatives (CFYDDI), situated in Wakiso District, central Uganda ${ }^{11}$. CFYDDI was co-founded by Dr Lawrence and Mr Magala in 2010. CFYDDI regularly work closely with local government and therefore the proposed clinics were discussed with the District Health Officer and the members of their District Health Team who expressed their 
support for this initiative.

Structure of the service: Advertising for the service was performed through school visits, local radio announcements and word-of-mouth. At presentation to the CFYDDI office, patients registered with a member of administrative staff before receiving a comprehensive oral hygiene educational module. The module was developed by the visiting dentist, Dr Barr, who used locally developed teaching aids, trialed with a small group of Ugandans to ensure local cultural practices were included. Patients then presented to Dr Barr where a thorough examination was performed, diagnoses were recorded, and treatments delivered as indicated, all with the assistance of a Ugandan translator. Dr Lawrence then administered post-operative care, prescribed any relevant medication, and applied fluoride where indicated, again with a Ugandan translator for assistance. Equipment was manually cleaned and then sterilised with bleach and Perasafe, an agent with a proven safety profile. There were sufficient policies for the management and disposal of clinical waste and sharps. Patients left the premises when they were deemed safe to do so, and those requiring restoration were referred to a local government clinic less than one mile away.

Treatment offered: Fluoride application for those 18 years and under, treatment of infection with antibiotics, simple debridement, extractions, and referral for restorations. Dr Barr also spent several days working at the local Health Centre where she observed, assisted and offered informal teaching to Ugandan trained dentists and dental officers in a clinical setting.

Funding: Equipment including the dental chair, examination equipment, elevators, forceps and dental syringes, was provided by a UK-based NGO: Dentaid ${ }^{12}$. Soft disposables, including local anaesthetic, topic fluoride, microapplicators, gauze, gloves and medicines, as well as sterilisation equipment and the costs of mobilising and remunerating local staff, were paid for through fundraising efforts of Dr Barr.

Ethics: All patients 16 years and over were assessed for capacity and those deemed to have capacity were seen and treated. Patients under the age of 16 all had written or verbal consent provided by a parent or guardian.

\section{Results:}


Three day long dental clinics were conducted over a two week period during October 2015. A total of 93 patients registered. 39 (42\%) were male, with an average age of 20 years (range 5-70 years). 27 patients were under the age of 16 , all of whom had parental consent. All adult patients had capacity. $86 \%$ of patients presented in pain. Diagnoses and treatments administered are summarised in Table 1 and Table 2 respectively. 26\% of patients were referred for restorations. There were no immediate complications associated with any treatment. A cost analysis was performed, bearing in mind that the only expenses were related to soft disposables, sterilisation equipment, medication, and mobilisation. The treatment provided equated to 178 Units of Dental Activity (UDAs). The total cost was $£ 169.46$ which calculates as $£ 1.82$ per patient, regardless of treatment, or $\mathrm{f0.95}$ per UDA. In Ugandan currency this equates to 8,700 Ugandan Shillings (UGX) per patient, regardless of treatment. Comparatively a patient would expect to pay 10,000 UGX for a restoration and 30,000 UGX for a single extraction.

\section{Discussion:}

We have demonstrated a definite need for dental treatment in this community and a clear organisational capacity to provide this treatment to high ethical and clinical standards. One key concern was regarding taking work away from pre-existing dental services: we found that government services which are supposed to be free impose a treatment charge and private dental clinics charge considerably more. Numerous patients were living with chronic pain, often for months or years, and despite the availability of services locally, they did not seek treatment. As this was the first time we had run this clinic we can safely conclude that they were not waiting for an international dentist to provide treatment. We can however strongly presume that costs were the main reason for this lack of health-seeking behaviour, a presumption that was based on anecdotal comments from patients and which is consistent with the findings of an in-depth qualitative study into barriers to seeking dental care conducted in Tanzania 4 . Similarly, we did not offer a superior range of treatments or use more advanced equipment than the local government and will not have created, nor perpetuated, the concept that DAOs can cause the treatments offered or equipment used by local providers to appear inferior.

Some aspects of the service were imperfect: we were only able to provide simple oral surgery and prevention which resulted in a significant number of patients being referred elsewhere for restorations. These restorations would incur a cost, a clear barrier to accessing treatment, and we 
could not ascertain if they sought this treatment after referral. Fortunately, we did not have any patients who needed complex oral surgery, requiring referral, a risk that was mitigated by the extensive experience of Dr Barr. The sterilisation process was pragmatic for the setting and resources available but was not as thorough as best practice in the UK, although we were not informed of any infection issues in the weeks following the clinics.

In terms of sustainability, the success of this project was wholly dependent on the loan of equipment from Dentaid, the clinical competence of the attending dentist and doctor, and the fundraising efforts of the team. Given that many patients did not present to local services, often for years, it would be pragmatic to continue providing such a service intermittently in the future. It is clear that strengthening local dental services and improving the capacity of Ugandan dentists and dental officers would be the optimal approach to this issue, but given the fact that these services incur a fee which clearly is beyond the financial capacity of the community, then we feel that our ongoing involvement can only be beneficial. From time spent in the local government clinic, it is clear that these services are oversubscribed, under-resourced, and affected by high levels of human resources for health absenteeism, and therefore additional capacity provided by the non-governmental sector can only help.

For such a project to be successful in the future it will be dependent on having professionals with the clinical and logistical experience partnering with proficient local people. We were fortunate to engage with an organisation well equipped to host our visit, and experienced in mass mobilization for health. Based on the clear need identified and the immediate impact shown by this feasibility study, it is our intention to continue with this work and to recruit additional volunteer dentists with the appropriate clinical experience in the future. In addition to the services we were able to offer, we would also hope to offer atraumatic restorative treatment. Alongside this, we intend to continue delivering the oral hygiene module through our existing links with schools and other NGOs as well as offering six-monthly fluoride application and providing fluoride toothpaste. All of this would result in the provision of the treatments outlined in the World Health Organisation's Basic Package of Oral Care ${ }^{13}$. One DAO that has made great progress is Bridge2Aid, a Tanzanian NGO with whom international volunteers provide theoretical and practical training to local healthcare workers and then supervise and monitor them in the clinical environment ${ }^{14}$. They have made significant advances in providing a sustainable Basic Package of Oral Care in Tanzania, particularly in rural areas, and this is a holistic approach from which we can learn a great deal. 
As key stakeholders in the local NGO with whom we worked, we feel a responsibility to become engaged in political lobbying to develop a strategy wherein there are adequate numbers of dentists and dental officers in Uganda with access to the necessary funds and resources. Until that point is reached however, we feel an obligation to work together to educate the local community, prevent dental disease, and offer a pragmatic clinical service in the meantime.

Beyond this single cultural context, there is a need for a paradigm shift in terms of international dental health. The discussion needs to be reignited and the arguments need to be reformed, developing the small body of (grossly repetitive) literature which currently exists, and increasing the volume of the voices demanding the right to oral healthcare.

If you are interested in learning more about this service, including potentially offering your clinical expertise, then please do not hesitate to get in touch with the authors.

\section{Acknowledgements:}

We are hugely thankful to the staff at CFYDDI for their help with both the organisation and the running of the clinics, particularly for translation services. Alongside this we are very grateful to the staff at DentAid for their loan of the equipment. Finally, we appreciate the local community for engaging with the project and providing vital feedback to the service.

\section{References:}

${ }^{1}$ Kassebaum NJ, Bernabé E, Dahiya M, Bhandari B, Murray CJL, Marcenes W. Global Burden of Untreated Caries: A Systematic Review and Metaregression. Journal of Dental Research 2015; 94(5): 650-8

${ }^{2}$ Robinson PG, Nalweyiso N, Busingye J, Whitworth J. Subjective impacts of dental caries and fluorosis in rural Ugandan children. Community Dental Health 2005; 22(4): 231-6 ${ }^{3}$ Listl S, Galloway J, Mossey PA, Marcenes W. Global Economic Impact of Dental Diseases 2015; 
94(10): 1355-1361

${ }^{4}$ Kikwilu E, Masala J, Kahabuka F, Senkoro A. Prevalence of oral pain and barriers to use of emergency oral care facilities among adult Tanzanians. BMC Oral Health 2008; 8:28

${ }^{5}$ Chher T, Hak S, Courtel F, Durward C. Improving the Provision of the Basic Package of Oral Care (BPOC) in Cambodia. International Dental Journal 2009; 59: 47-52

${ }^{6}$ Ministry of Health. Health Sector Strategic Investment Plan 2010/11 - 2014/15. Kampala:

Government of Uganda; 2010

${ }^{7}$ Ntulume D. Oral health should be given priority. 2009. Online information available at http://www.newvision.co.ug/D/8/459/702188 (accessed November 2015).

${ }^{8}$ Benzian H, Gelbier S. Dental aid organisations: baseline data about their reality today. International Dental Journal 2002; 52(5): 309-14

${ }^{9}$ Dickson M, Dickson GG. Volunteering: beyond an act of charity. J Can Dent Assoc 2005; 71(11): $865-9$

${ }^{10}$ Han SJ, Quiñonez CR. Dental Volunteerism: Is the Current Model Working? J Can Dent Assoc 2013;79:d69

${ }^{11}$ CFYDDI. The Centre for Youth Driven Development Initiatives. 2015. Online information available at www.cfyddi.org (accessed November 2015).

${ }^{12}$ Dentaid. Improving the world's oral health. 2015. Online information available at www.dentaid.org (accessed November 2015).

${ }^{13}$ Helderman W, Benzian H. Implementation of a Basic Package of Oral Care: towards a reorientation of dental NGOs and their volunteers. Int Dent J 2006;56(1):44-8

${ }^{14}$ Wilson K, Wilson I, Holmes R. Oral urgent treatment (OUT) - a volunteer led training programme in North West Tanzania. British Dental Journal 2012; 212(9): 443-8

Table 1: Diagnoses of patients (some patients had multiple diagnoses)

Diagnosis

Sound teeth

Sensitive teeth
Number of patients

$5(5.4 \%)$

$10(10.7 \%)$ 


\begin{tabular}{lc}
\hline Gingivitis & $5(5.4 \%)$ \\
\hline Periodontitis & $3(3.2 \%)$ \\
\hline Pericoronitis & $1(1.1 \%)$ \\
\hline Pupillary necrosis & $1(1.1 \%)$ \\
\hline Caries & $22(23.7 \%)$ \\
\hline Gross caries in one tooth & $35(37.6 \%)$ \\
\hline Gross caries in two teeth & $5(5.4 \%)$ \\
\hline One retained root & $3(3.2 \%)$ \\
\hline Two retained roots & $3(3.2 \%)$ \\
\hline Three or more retained roots & $1(1.1 \%)$ \\
\hline Mouth ulcers & $1(1.1 \%)$ \\
\hline Left before being seen & $1(1.1 \%)$ \\
\hline
\end{tabular}

Total patients: 93 , some had multiple diagnoses. 
Table 2: Treatment delivered (some patients had multiple treatments)

\begin{tabular}{ll} 
Treatment & Number of patients \\
\hline Single tooth extraction & $35(37.6 \%)$ \\
\hline Double tooth extraction & $7(7.5 \%)$ \\
\hline Triple tooth extraction & $1(1.1 \%)$ \\
\hline Declined extraction & $7(7.5 \%)$ \\
\hline Hand scaling & $2(2.2 \%)$ \\
\hline Paracetamol & $43(46.2 \%)$ \\
\hline Amoxicillin & $7(7.5 \%)$ \\
\hline Metronidazole & $1(1.1 \%)$ \\
\hline Fluoride & $65(69.9 \%)$ \\
\hline OHI & $92(98.9 \%)$ \\
\hline Smoking Cessation Advice & $1(1.1 \%)$ \\
\hline Did not attend & $1(1.1 \%)$ \\
\hline
\end{tabular}

